\title{
Find and Advertise Jobs for Mathematicians at MathHire.org
}

Daniel Lütgehetmann and Sebastian Meinert (both Freie Universität Berlin, Germany)

The World Wide Web now hosts a new website for jobs for mathematicians, launched earlier this year at MathHire.org. In collaboration with professors, graduate students and university administrations, a new tool has been designed, programmed and tested that smoothly connects employers and jobseekers in mathematics.

We knew about 'mathjobs' offered by the AMS and other existing websites but we wanted to give the concept a complete overhaul and significantly improve the user experience. After months of hard work, we are convinced that MathHire leaves little to be desired: job listings are published in quick time, the job search is highly intuitive and the platform is finely crafted with attention to detail.

In the current phase, we are primarily focusing on the European market but if the tool is well received, we will strive to expand our services.

Universities and research institutions may publish advertising-only job listings free of charge but the system delivers its full strength to employers who accept applications through MathHire.org for a fee. Employers then benefit from a sophisticated web interface to review and evaluate received applications (see https://mathhire. org/showcase). Letters of recommendation are confidentially managed through MathHire. And last but not least, employers may establish a digital hiring committee and

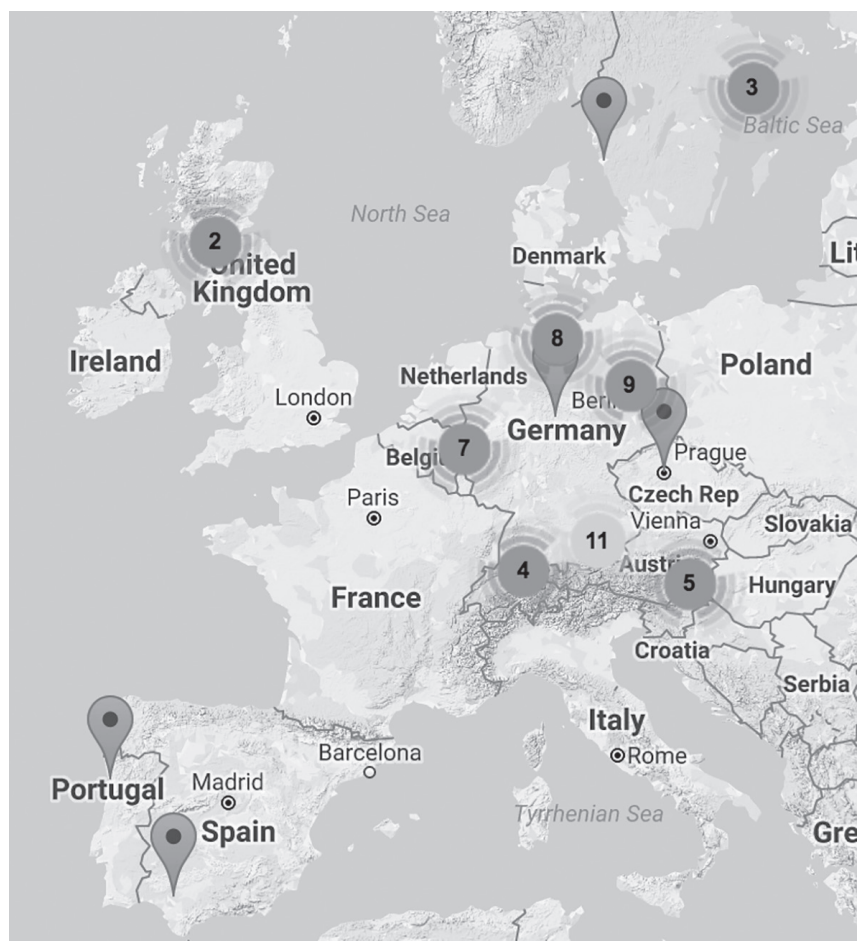

grant colleagues the right to review and evaluate applications in teams.
In cooperation with

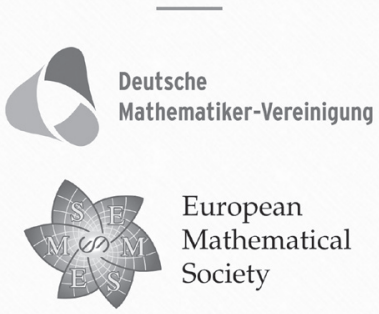

After initial test runs and collaboration on central features of the platform, the EMS has signed a cooperation agreement with MathHire to help the new website to grow. As part of the cooperation, the institutional members of the EMS receive one free listing per year and a $20 \%$ discount on every paid listing thereafter. In order to claim these benefits, representatives are asked to visit https://mathhire.org/ems and follow the instructions.

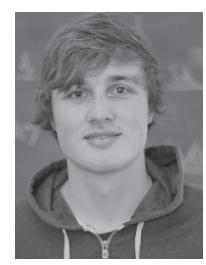

Daniel Lütgehetmann [daniel@mathhire. org] is a graduate student in mathematics at Freie Universität Berlin. He is studying Teichmüller spaces under the supervision of Holger Reich.

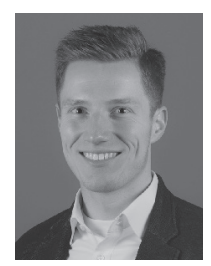

Sebastian Meinert]sebastian@mathhire. org] studied mathematics at Freie Universität Berlin, where he received his PhD in 2014. He studied deformation spaces of trees, also under the supervision of Holger Reich. 